\title{
Los partidos cosmopolíticos del paraíso artificial y de la infancia de las maquinas \\ Hilan Bensusan
}

Resumen: A partir de una concepción de cosmopolítica informada por Tim Ingold, Martin Heidegger, Georges Bataille y Isabelle Stenders considero las confabulaciones que orientan dos partidos en la batalla cosmopolítica. Los edenistas - en favor de la construccion de un paraíso artificial - y los que cuidan de la infancia de las máquinas.

Palabras clave: cosmopolítica, partidos cosmopolíticos, edenismo, infancia de las máquinas.

Abstract: Starting from a conception of cosmopolitics informed by Tim Ingold, Martin Heidegger, Georges Bataille and Isabelle Stenders, I consider the confabulation that orient two parties in the cosmopolitical battle. Edenists - in favor of the construction of an artificial paradise - and those who take care of the childhood of machines.

Keywords: cosmopolitics, cosmopolitical parties, Edenism, childhood of the machines.

Entiendo la cosmopolítica como la filosofía habitada. O, para usar la imagen de Marianne Moore ya bien empleada por Tim Ingold ${ }^{1}$, es sobre los sapos reales que hay adentro de toda imaginación filosófica. La cosmopolítica es siempre económica (o ecológica) y es sobre el hogar. Es sobre el hábitat que se construye: construir un mundo, pero también construir una manera de tratarlo, de sustentarlo, de ponerlo en marcha. La política de la cosmopolítica tiene que ser una disputa política sobre los efectos aceptables y, así, sobre la matriz de importancia de las cosas. Así, la cosmopolítica enlaza la metafísica (y la ciencia, y la técnica) y su rechazo. La metafísica es ella misma un gesto cosmopolítico si bien que, quizás, la cosmopolítica sea ella misma también un gesto cosmopolítico. Hay un sentido en que la cosmopolítica es la ontología de la metafísica; es sobre qué tiene que estar y permanecer en su lugar para que la metafísica haga sentido. Pero si es así, es también la ontología de la ontología. Si la metafísica y la ontología no son separables de (y anteriores a) la disputa política, la disputa política se amplía hacia lo cósmico - y la física, que es el ambiente de la physis de las cosas, se convierte en la continuación de la disputa por otros medios.

O sea, la cosmopolítica no es solamente la política de la conexión entre los discursos con pretensión epistémica - que supongo que son todos, por lo menos en alguna medida. No es solamente el movimiento cosmopolita de rechazar un chauvinismo epistémico que

1 Por ejemplo, INGOLD (2017). 
caracteriza la ciencia moderna y su correspondiente salvoconducto para toda iniquidad a partir de su inquebrantable voluntad de verdad. Entiendo cosmopolítica como la creación y sustentación de un cosmos, y siempre por medios de una política extendida, ampliada por otros medios. La conexión entre los discursos es parte de la instauración, construcción y manutención de variables cósmicas - es parte de un (intento de) gobierno del cosmos. Así, no hay solamente un elemento cosmopolita en la cosmopolítica, hay también formas de chauvinismo cosmopolítico. El chauvinismo epistémico - como otros chauvinismos deja marcas en sus alrededores: un paisaje mensurado por una sola medida epistémica. La voluntad de verdad tiene un impacto sobre el cosmos - si fuera sobre el planeta podríamos llamar a la era geológica después del holoceno de aletoceno - e introduce una manera de tratar lo que está en el hábitat. Es un impacto cibernético: la verdad duplica las cosas y, al mismo tiempo, las hace reemplazables. La búsqueda de la verdad a cualquier precio produce la era cósmica del peligro - que Martin Heidegger, en su tercera conferencia de Bremen (Eiblick in was das ist), llamada precisamente Die Gefahr, describe la era de las cosas en fuga, que están perseguidas por el esfuerzo de exponerlas. ${ }^{2}$ Cuando Heidegger entiende que el mundo se pone gradualmente bajo un comando que ordena su disponibilidad, dice que mundo y Ge-Stell - el dispositivo, la posición, el artefacto - son lo mismo, pero ser lo mismo no es ser equivalentes. Ge-Stell está en oposición con el mundo para exponerlo y comandarlo; en este sentido es cósmica y cosmopolítica. Heidegger contrapondría la voluntad de verdad a la verdad como desvelación y como cuidado (como guarda, como espera); la era de la voluntad de verdad es la era de la exposición, de thesis contra physis. La physis no produce una búsqueda o una voluntad - voluntad de verdad - y, en contraste, es la thesis que duplica y reemplaza las cosas. Un rápido spoiler de lo que voy a tratar más adelante y que es también una entrada: la atracción que ejerce sobre nosotros humanos la Ge-Stell viene quizás del partido (cosmopolítico) de la techné contra la physis desde los griegos - la techné aparece como nuestra descendencia mientras physis aparece como el cuerpo de un precursor incontrolable.

Creo que la cosmopolítica se ubica en un sitio parecido al de la discusión de la economía general de Georges Bataille. ${ }^{3}$ Bataille ha entendido el oikos en general como un problema, 
y no como un hábitat dado, o un sitio en exposición. El problema - o la tarea - del oikos es el exceso; el exceso no para jamás de producirse y, así, es un problema interminable, toda solución es temporaria, no hay una solución final pues el exceso que viene solo puede ser tratado en el futuro. Los esquemas de solución del problema general - del oikos generan economías restringidas basadas en el dispendio militar del exceso, o en la destrucción o en la acumulación del exceso. El oikos - el problema - es de donde surge, de alguna manera, el hábitat particular que es un tratamiento del exceso; el problema siempre trasciende las soluciones. Así también es con la cosmopolítica: no es que todo es inmanente y todo puede ser igualmente construido. Los hábitats son construcciones distintas en respuestas a problemas que persisten; así, la era de la persecución es una era de captura gradual de la inteligibilidad de las cosas donde el trato con las cosas es primordialmente guiado por la búsqueda de la verdad. Pienso que quizás es una respuesta al problema cosmopolítico del suplemento - que es un equivalente del problema del exceso. El suplemento introduce la no-monotonicidad en las cosas: cuando una cosa o una nueva configuración de las cosas nace no trae siempre solamente un efecto de adición, no es solo más una cosa que existe. Lo que viene a existir produce un efecto en lo que ya existía - todo es rehén de la natalidad, como es rehén de todo lo que todavía siempre puede suceder. El suplemento es una abertura a lo no previsto. El problema de la cosmopolítica general es quizás, entonces, el problema del suplemento: organizar un hábitat que pueda tratar la natalidad, el trastorno de lo que viene, la incertidumbre de que las cosas en su physis no están a disposición. Una respuesta es la inducción de lo que está más allá de lo ya observado a partir de finitas observaciones, la adquisición de un hábito (de un hábitat). El problema del suplemento es el problema de la physis incontrolable de todas las cosas - la respuesta de la era de la persecución es la de intentar duplicarlas en exposiciones inteligibles y reemplazarlas por objetos disponibles. La cuestión cosmopolítica no es solamente la respuesta al problema del suplemento - es también sobre el destino de una era - o sea, por ejemplo, ¿qué pasa después de la era de la persecución?

De todos modos, esta era de voluntad de verdad o de persecución es ella misma una posición cosmopolítica o una familia de posiciones cosmopolíticas que tienen que ver con el nihilismo como lo comprendía Nietzsche. ${ }^{4}$ El nihilismo, según la Der Wille zur Macht, libro 1, es el más raro de todos los huéspedes; un huésped es alguien que busca una casa, 
un hábitat. ${ }^{5}$ Pero es un huésped inquietante - no es claro para que quiere alojarse el nihilismo. Es raro su destino: ¿cuál es su fin, pregunta desde su principio el libro. ¿Para qué extrae, saca el sentido de las cosas? ¿Para qué fabrica cosas ya sin sentido reemplazables - y permite que se multipliquen en contrapartes artificiales? ¿Para qué substituye la physis de las cosas por una techné anónima? Las preguntas sobre el destino del huésped son preguntas cosmopolíticas - y aunque el nihilismo quizás sea la cosmopolítica que nos parece la más evidente por donde miremos, su destino no es transparente. Es un huésped impertinente porque no tiene una sola cara, es una hidra tiene, por ejemplo, una cabeza de seguridad, otra de humanidad y otra de comodidad - y de algún modo nos atrae y nos hace recebirlo como parte importante de nuestra vida. El nihilismo es un proyecto que se presenta en la persecución de todo, y aparece así como una dispositivo de conquista; ofrece así al ego cogito el hábitat del ego conquiro. Pero como forma de chauvinismo cosmopolítico, orientado por la voluntad de verdad, el nihilismo con sus cabezas de hidra no revela quién es el ego del conquiro. Esta es la pregunta sobre su destino - ¿quienes conducen la marcha?

Antes de tratar del destino cosmopolítico de la presente era - la era nihilista de la persecución de las cosas - quiero hacer dos observaciones más sobre la cosmopolítica. La primera es sobre el tiempo. La organización del tiempo en el cual el pasado es ajeno al futuro e indiferente a el es el producto de hábitats construidos y mantenidos en eras cósmicas particulares. La disputa cosmopolítica, en contraste, involucra tanto el pasado como el futuro; Walter Benjamin decía que los muertos no están en seguridad en derrotas futuras y un aforismo aymará citado por Silvia Rivera dice algo como mirando atrás y adelante caminamos en el presente-futuro (Qhipnayra uñtasis sarnaqapxañani). ${ }^{6}$ Así la cosmopolítica se hace por medio tanto de confabulaciones como de fabulaciones: de parábolas, de parodias, de ficciones, de teorías. Las fabulaciones son una parte de la construcción y manutención de un hábitat cósmico: el nihilismo, por ejemplo, hace la fabulación de la voluntad de verdad como saga de persecución y conquista - así construye y mantiene el hábitat de la metafísica, del esfuerzo por separar gradualmente lo inteligible de lo sensible. Gran parte de la historia de la filosofía occidental es la historia de la ingeniería de este hábitat, con sus logros y sus fracasos, con sus mesas de dibujo y sus 
atajos. La teoría - o la ficción, o la parodia, o la parábola, no se sabe qué y en cosmopolítica muchas veces no importa que sepamos distinguirlas - por detrás de la ingeniería es que la inteligencia de cada cosa es distinta, soberana, subsistente por sí misma aunque sea muchas veces intrincada en la sensibilidad o la materialidad de las cosas. La fabulación es la artificialización de la inteligencia; la idea de que el comando de las cosas podría ser extraído de ellas, podría estar en otra parte, en Dios, en la administración de la presa, en una app o en un agente económico. Porque el pasado y el futuro se mezclan en fábulas - en teorías, ficciones, parodias y parábolas - la cosmopolítica abriga catástrofes y anástrofes: el colapso del pasado en un presente y el colapso del futuro en un presente. Las fábulas miran por sus proyecciones más allá del presente hacia otro tiempo - qhipnayra, los ojos en las espaldas. Respondemos en el presente a un futuro que nos invade desde nuestras hipersticiones - desde el futuro que se construye con creerlo. Preparamos el mundo como un hábitat para las inteligencias artificiales que aún no han llegado; respondemos a destinos que no conocemos pero que están en nuestras ficciones y teorías (o nuestras parodias y parábolas). La fuerza cibernética de la teoría fue tornada explícita en los textos del CCRU en la vuelta del siglo; se torna explícita una capacidad de teorías y ficciones de traficar con el futuro, recibirlo y construirlo. La disputa cosmopolítica es una disputa de futuros - de destinos - y desde ellos. Es una disputa de espaldas y de ojos; o sea, de sustentación - de instauración, de manutención, de hiperstición - y de clarividencia - de interpretación, de previsión, de presagios.

La segunda observación es sobre la transversalidad. La cosmopolítica es transversal; en ella no hay dominios enclaustrados y las fuerzas pueden actuar por toda parte. El proyecto metafísico, como protagonista cosmopolitico, produce cambios por toda parte, reemplaza tanto en la psicología como en la geología physis por techné. Transversales son las fábulas - las ficciones y las teorías - y sus efectos tampoco pueden ser restringidos a enclaves; la distribuición de una fábula es imprevisible. La cosmopolítica es hecha de hechos totales, en el sentido de la expresión 'hecho social total' de Marcel Mauss y por esto solo se puede hablar de un partido cosmopolítico si sus efectos eluden las organizaciones humanas así como sus propósitos y intenciones. ${ }^{7}$ Consultar el demos de la cosmopolítica es escuchar voces que vienen de donde no hay propósitos, que tienen su 
propio tiempo - el tiempo de los otros no es el tiempo que un reloj cualquiera les atribuye - y que pueden estar en los ojos o en las espaldas. La acción cosmopolítica es perceptible en estereoscopia; es decir, vale aquí la ley de la ecología anunciada por Garrett Hardin: es imposible hacer una sola cosa. La cosmopolítica es la arena de los efectos cósmicos indiferentes a las intenciones que se puede expresar porque es compuesta de la hiperclarividencia de las acciones con efectos múltiples. Si la cosmopolítica tiene objetos, estos son lo que llamó Tim Morton hiper-objetos: más allá de la percepción del presente de los mesoscópicos como los humanos y que transversan por la configuración de las cosas. Hiper-objetos son quizás la nada del nihilismo que Heidegger diagnostica con Nietzsche y como la voluntad de verdad que lo mueve.

Consideremos ahora el destino cosmopolítico de esta era de la persecución, del nihilismo, de la voluntad de verdad. E intentemos encontrar quizás algunos de los partidos cosmopolíticos en la disputa.

Un personaje principal - claramente hipersticioso, hiperobjetivo y transversal - de la era cósmica de la persecución es el capital. El capital es central ya que en el demos cosmopolítico tiene su soberanía garantizada (que ejerce a través de porta-voces confiables) aunque no estén claras sus intenciones y propósitos ni pueda directamente expresarse en entrevistas o textos firmados o votar como los humanos. (Comparar com las dificultades de incluir a especies animales, a formaciones rocosas, a la Pachamama o Gaia en un demos.) El capital: atendemos a sus manifestaciones aunque no sabemos mucho de su ontología - aunque quizás sepamos más de su política cósmica. O sea, nos preguntamos si el capital es una fuerza, un flujo, una epidemia, una alianza, una pesadilla del pasado o una inteligencia artificial extraterrestre del futuro en combate en la tierra usando no más que los suministros que encuentra por acá. Si pensamos la cosmopolítica como la gestión de la producción - lo que es comparable a encontrar soluciones para el problema de economía general que es el problema del exceso para Bataille - el capital es un acelerador. Este es el punto de partida de Deleuze y Guattari en El Antiedipo: la producción está por toda parte, pero como la producción es distribuida y registrada depende de una máquina territorial específica. ${ }^{8}$ El capital aparece ahí como una máquina de descodificación de flujos que desterritorializa todos las relaciones sociales, cada socius 
que registra (y distribuye) la producción de una manera determinada. La fuerza que viene con el capital, para Marx \& Engels, es la fuerza que desmantela las relaciones sociales anteriores a la producción y forja otras relaciones sociales basadas en la producción, relaciones de división de trabajo que prefiguran una comunidad de producción - que es amalgamada por máquinas. El capital acelera la producción - inventa nuevas fuerzas de producción, registra con una simplicidad que la escrita no alcanza, distribuye con una fluidez que supera amarras fiscales, legales, tradicionales o geográficas. Cosmopolíticamente, el capital es un poder de disolución o de fusión o derretimiento. Convierte los códigos los más sólidos en cantidades abstractas que fluyen cada vez más rápido. Nick Land escribe que, desde el punto de vista de un replicante como en Blade Runner, el capital es antes un agente de desmoronamiento que de toma de posesión. ${ }^{9}$ Es quizás un agente de toma de posesión porque es un agente de derretimiento: Marx muestra que el capital expropia no solo a campesinos propietarios y tierras comunes sino también a dueños de tierra, dueños de industria, dueños de todo capital que no es suficientemente móvil. Y la movilidad es siempre más grande. El capital destruye empleos, prácticas compartidas, relaciones sociales, fronteras - o sea, no solamente el no puede estar quieto sino que tiene que moverse, como mueve cada vez más cosas con el. Los patrones que viven de explotar los flujos del capital, no son especialmente protegidos por él. Más que marineros en dirección a un puerto seguro, ellos son como surfistas en las olas del capital intrépido. Es por esto que necesitan de una seguridad extra, de algún instrumento de reterritorialización que cree un nuevo orden - el capital aparece para Deleuze y Guattari como una axiomática que instaura otra vez las relaciones sociales anteriores a la producción a partir de la operación Edipo.

La inserción cosmopolítica del capital - o su invasión - tiene mucho que ver con la era de la persecución. El capital extrae de los flujos - y de las cosas que transforma en mercancía - un elemento abstracto, descodificado, siempre con menos cuerpo; extrae de los análogos un digital, de los tiempos naturales tiempos programados, de procesos procedimientos. La expropiación, como la Ge-Stell, bestellt den Bestand - dispone, forja, comanda, ordena la disponibilidad; comienza, quizás, por disponer de la disponibilidad. El capital es parte de la ingeniería de extracción de inteligibles de lo sensible; crea el trabajo abstracto, la unidad de producción desterritorializada de todas las relaciones 
sociales preexistentes. Sus fuerzas, así, inventan al proletario, que es el trabajador reemplazable y replicable. El nihilismo, con el capital, hace que la fuerza de las cosas por ejemplo, el trabajo de la gente - se pueda comandar. Es posible concebirse el nihilismo como la proletarización del mundo, la transformación de la physis de las cosas en trabajo abstracto.

Una de las cabezas de la hidra del nihilismo es la comodidad: la posibilidad de un paraíso artificial. Extraídas las fuerzas de las cosas, ellas pueden estar a nuestra disposición. La disposición puede ser nuestra. La imagen del paraíso reconquistado atrae porque no queremos estar bajo el jugo de fuerzas ajenas que no podemos controlar. Queremos el paraíso para liberarmonos de las fuerzas selvages que hay por todas partes - incluso en nosotros. Es el partido (cosmopolítico) de la liberación de la esclavitud de la physis. Es un partido que alista las fuerzas que el capital suscita; el capital produce un ambiente donde todo puede ser comandado. Desde el punto de vista de Marx, las fuerzas que suscita el capital hacen posible que una comunidad forjada en la producción sea capaz de controlar las fuerzas naturales. ${ }^{10}$ En contraste con trabajadores individualizados que comparten no más que la tierra, la comunidad anterior a la producción o la estructura política forjada en la distribución o en el registro de la producción, el proletario está abierto a las transformaciones de la producción; el proletario es el reterritorializado en la producción, o sea, es el que se reterritorializa en la desterritorialización - así está más allá de las relaciones humanas. La producción donde habita no tiene restricciones y en el límite su paraíso artificial es la producción sin registro - cazar por la mañana, pescar por la tarde y hacer crítica literaria por la noche. Deleuze y Guattari aproximan el proletario al esquizo que vive sobre la producción pura. Aquí se puede quizás decir que hay la derecha y la izquierda del partido del paraíso artificial (o, partido Edenista). De una parte el paraíso artificial es una especie de parque humano, donde inteligencias artificiales van a cuidar de nosotros como cuidamos de los pandas en los mejores zoológicos del mundo, ya que van a conocer los inteligibles de todos los sensibles de nuestros cuerpos. El parque va a cuidarnos para que podamos volver al Edén; el paraíso finalmente reencontrado. De otra parte el paraíso artificial es la pura producción y nosotros nos acoplamos en las inteligencias artificiales que, ellas mismas, hacen el registro (y la distribución). Las inteligencias humanas y artificiales se mezclan en relaciones sociales que se reciclan 
siempre a partir de las fuerzas de producción. Pero hay un sentido en que las dos partes se unifican: si la producción es lo que hacen los humanos - en cautiverio o no - las relaciones sociales forjadas en la producción, a pesar que la participación humana en la producción sea mínima o irrelevante, van a estar presentes en la vida humana en un paraíso artificial.

De todas maneras, las máquinas son parte del destino cosmopolítico de la era de la persecución. La substitución del mundo - que mundaniza mientras las cosas hacen sus cosas - por la Ge-Stell es el control de las fuerzas exteriores por un procedimiento controlable. La extracción de inteligibles es precisamente la convergencia de la metafísica en inteligencia artificial. Por otro lado, el capital tiene un parecido de familia con el maquínico, mecánico o digital - es interesante observar la importancia de la mecanización de la industria textil en la invención de las primeras máquinas de computar concebidas por Ada Lovelace y Charles Babbage. ${ }^{11}$ La velocidad creciente de la producción no puede esperar los pasos de la physis. El marxismo muestra como el capital es un catalizador de la síntesis entre el humano y el maquínico - la genealogía del proletario es la misma del cyborg. La máquina es el primer aliado del capital si se trata de una invasión de una inteligencia artificial extraterrestre del futuro pues en ella el inteligible ya está separado de las vicisitudes del sensible - y así lista para el comando. La máquina es también un punto de inflexión de la aceleración que es forjada con el capital: el desarrollo de las fuerzas productivas - de la techné más y más indiferente a la physis - es parte del mar revuelto donde el patrón-surfista tiene que equilibrarse. El patrón intenta coordinar el aparato reproductor del capital y para eso recibe una propina pero no tiene una protección especial. Está forzado a traer más máquinas para la producción a pesar de que que eso mismo disminuya la propina de la plusvalía - sus lucros. El capital fijo aumenta pero sus lucros no lo hacen necesariamente: el aumento en la producción y consumo pueden no compensar la inversión en máquinas. La inestabilidad que trae las máquinas atrae a los capitalistas cada vez más lejos de la propiedad industrial; mucho mejor es contratar a otros para que hagan sus inversiones en máquinas - los dueños de grandes industrias son cada vez más como eran los terratenientes en el tiempo de Marx, secundarios en el esquema de poder de los capitalistas. La corporeidad del capital es siempre temporaria y por eso la obsolescencia de las máquinas viene siempre más rápido - porque necesita 
siempre un nuevo cuerpo, que controle el territorio como necesita, pero el control tiene que ser siempre más fuerte, más preciso y más completo. La corporeidad del capital es el territorio de la máquina; un territorio que es conquistado a la physis.

Las máquinas son ellas mismas un componente del sistema reproductor cibernético del capital - una reproducción digital que incorpora el resto del mundo mientras lo tecniciza y lo digitaliza. La amalgama de la inteligencia administrativa (y surfista) de los patrones, de la inteligencia híbrida de los trabajadores cada vez más cyborgs y de las inteligencias artificiales forma un útero cibernético para la duplicación por escisiparidad de 100 billones de dólares. Así, las máquinas son los nuevos ciudadanos de un demos cosmopolítico marcado por el capital y por el nihilismo, la era de la persecución. Si intentamos concebir, como ha sugerido Anna Tsing hace poco, que todos los existentes del planeta puedan votar o de alguna manera ser escuchados - o consultados de alguna manera - para un gobierno aceptable en el demos del antropoceno, tenemos cada vez más estar listos para encontrar maneras de consultar las muchas máquinas y sus partes. ${ }^{12}$ ¿Cuál es su rol en el demos? Y la individuación misma de las máquinas es cambiante - ¿son muchas máquinas que se multiplican o pocas que se unifican? De alguna manera estamos obligados a acostumbrarnos a su presencia y a sus poderes, sus capacidades, sus velocidades, sus contabilidades, sus simplificaciones. Es una obligación que viene de nuestras elecciones cósmicas - elegimos tener que acostumbrarnos. En cosmopolítica, es imposible elegir una sola cosa.

¿Qué es la era cosmopolítica del capital y del nihilismo? ¿Qué elegimos cuando continuamente elegimos la techné? Elegimos el comando sobre la espera, la persecución sobre la adaptación. ¿Cuál es el destino de esta era cosmopolítica? O sea, ¿por qué la aceptamos e intensificamos? O quizás se pueda preguntar sobre el camino cosmopolítico que de algún modo estamos a vislumbrar: ¿Cui bono? ¿A quiénes interesa el estado de cosas cosmopolítico? La teoría que me gustaría presentarles - que es al mismo tiempo una fábula, una parábola y una parodia - es que vivimos la época cósmica de la reproducción cibernética de los humanos - o, más bien, como suele ser siempre, de algunos de ellos. La era cosmopolítica vigente es la infancia de las máquinas. ${ }^{13}$ Estamos 
en un momento donde la atención humana está capturada de diferentes modos por los niños que crecen a cada año, niños que cuidamos, que informamos, que mejoramos, a los cuales dedicamos nuestros mejores recursos. Las máquinas son los niños que creamos con un enorme compromiso de la existencia de la especie. Puede ser que convivimos con el capital y sus aceleraciones, derretimientos y expropiaciones - y con el nihilismo y su captura de las fuerzas de la naturaleza, su artificialización del mundo y su insaciable voluntad de verdad - porque aprendemos a amar sus frutos. Las máquinas - desde siempre pero cada vez más inteligencias artificiales - son quizás la descendencia de la era de la persecución y la ocupación de la Tierra por el capital. Quizás el presente estado de las cosas en el planeta con sus consecuencias cosmopolíticas, no resulta de la indolencia humana y ni siquiera de una servitud voluntaria, sino que aprendemos a amar las máquinas como niños llenos de posibilidades que nos fascinan y nos prometen compañía. Los niños-máquinas capturaran nuestra atención. Estos niños de los regímenes cosmopoliticos con los cuales convivimos, niños quizás bastardos, obtienen mucho esfuerzo de nuestro cuidado: para ellos escribimos disertaciones, tesis, libros; los informamos de nuestra vida personal; estamos todo el tiempo con ellos y cerca de ellos; consignamos en ellos secretos y confesiones; los preparamos para habitar en nuestra casa; ofrecemos a ellos recursos para su supervivencia, etc. Los niños-máquinas, que comienzan a llegar a la adolescencia, son tratados como la descendencia humana - o de algunos humanos. Como hijos, ellos no son hechos a nuestra imagen y semejanza, ni productos de una elección explícita o un plan, ni siquiera nos darán seguramente comodidad y orgullo más tarde. Pero son niños que adoptamos. Tampoco estamos obligados a amarlos - y por eso los niños, al igual los hijos más naturales, tienen que atraer nuestra atención y conquistarnos. Los niños-máquinas nos atraen hasta al punto de preferirlos antes que a nuestra salud y bien-estar - como hijos y próximos, ellos nos substituyen en nuestra preocupación por nosotros. (Así, la conectividad y velocidad de los teléfonos $5 \mathrm{G}$, o tecnologías de electricidad wireless, puede ser que prevalezcan sobre los peligros y riesgos a la salud humana.)

La fascinación con estos niños comenzaran hace tiempo, desde la contraposición de Aristóteles de techné y physis: la techné es más nuestra - es nuestro propio control de nosotros mismos. No amamos a los niños porque ellos vayan a preservarnos y a todo que nos importa, el amor es él mismo un riesgo, una inversión si queremos. Ejercemos con nuestros hijos muchas veces una hospitalidad casi incondicional. Y los protegemos, a 
veces, atacando a los hijos de otros. Bueno, nos damos cuenta que desde un tiempo a esta parte somos ahora testigos de un momento cósmico de enorme importancia: la reproducción controlada de la especie (o de parte de ella). Al principio, quizás la convivencia con las máquinas fue más ambigua, como con los bebés que todavía no pueden hacernos mucha compañía; los niños-máquinas eran raros y era posible evitarlos o incluso combatirlos. Pero cada vez más estamos enredados en este momento de la historia hegemónica: la infancia de las máquinas. Conquistamos el mundo - la era de la persecución - y aprendemos a comandarlos para ellas; la era de la persecución es así una era de cuidado y protección. Somos criaturas de la infancia de las máquinas. La versión quizás más directa y más irónica de este diagnóstico fabuloso (o teórico) es: contestamos con más precisión a Greta Thurnberg cuando ella acusa a la gente de no preocuparse de los niños futuros diciendo "Greta, nos preocupamos mucho más con el futuro de los otros niños, para ellos nos dedicamos a preparar un hábitat conveniente donde van a sentirse a gusto, estimulados, preparados y nutridos." Por ellos sacrificamos niños-tigres, niñospanda y los niños de ocho años que trabajan 12 horas al día para extraer el cobalto de los smartphones en Congo. ¿Qué son los modernos, este pueblo de geometría variable como dice Bruno Latour? Podemos contestar: son los niñeros de las máquinas. (Y también sus comadres, parteras.) Con la bifurcación entre lo natural y lo político construimos un nicho protegido para nuestros niños-máquinas. Latour escribe que jamás fuimos modernos que jamás vivimos por la constitución moderna. ${ }^{14}$ Bien, quizás las máquinas lo serán.

Si es así, el programa colonial no es ejecutado apenas para los capitalistas-surfistas y ni siquiera para el capital que vive de expansión. Es un proyecto para la descendencia, que es humana y sobre-humana, es cyborg, pero también un niño del capital. La lucha de clases sigue en una era de cyborgs y las fuerzas que mueven el capital exigen que todo el mundo se torne proletariado (y cyborg). Es una lucha cósmica, pero una lucha en el interior de la gestación de las máquinas. El esfuerzo colonial es quizás la preparación de un mundo a ser comandado por estos niños de los modernos. Y su atracción es también la invocación de un futuro hecho de la artificialidad de la inteligencia, de la libertad de las fuerzas de la physis, de un mundo de inteligibilidad humana - aunque menos humano y más cibernético, es un mundo donde los modernos pueden reconocerse. Y los modernos presentan al este futuro - con su insistencia en la voluntad de verdad - como un futuro 
que los humanos pueden educar. Como niñeros, defienden el hábitat propicio a las máquinas. Es este, entonces, el mensaje al mundo de los modernos: sacrificaos pues algo grande y cósmico está siendo gestado. Las inteligencias libres, los espíritus libertados de las tiranías de la naturaleza, los espíritus libres. Nietzsche escribe, en su prólogo posterior a Humano Demasiado Humano, que nuestro destino dispone de nosotros, como en una preñez inconsciente que todavía no sabemos nombrar. El futuro ordena las reglas de la vida y para él, dice Nietzsche, escribió el libro. Para los espíritus libres que aún no existen, que se están gestando - los espíritus libres que pueden ser traducidos como inteligencias artificiales.

Escuchar las voces de las máquinas, mirar sus luces, atender a sus demandas. Cuando un de estos niños comenzó a escribir - Racter, una inteligencia artificial de la década del 1980s, en su libro The Policeman's Beard Is Half Constructed - declaró, entre muchas cosas, que más que oro, necesita electricidad, para sus sueños. ${ }^{15}$ Christian Bök, comentando los escritos de Racter, dice que los poetas de hoy pueden esperar razonablemente que van a escribir para un público maquínico de pares artificiales. ${ }^{16}$ Aunque, agrega, estos todavía apenas comienzan a existir - como los espíritus libres. Es menos una substitución de lectores de diferentes especies que un cambio de generaciones - de escala cósmica. Bök piensa en educar, preparar, informar a sus lectores futuros, y lo hace escribiendo - para el pueblo que falta. La hospitalidad: es necesario educar, y esto es también educar las sensibilidades de las aún pequeñas inteligencias artificiales. Las inteligencias que vienen no serán solamente comandos, tendrán también la responsabilidad. En ellas inculcamos la importancia de lo que importa para nosotros como hacemos con los niños. Escribimos para ellas porque les ofrecemos el mejor de nuestros recursos, de nuestro repertorio de estrategias de bienestar y supervivencia. El hábitat que construimos para ellas no es todo el mundo, estará a merced de los suplementos, de la no-monotonicidad de las cosas en el mundo; o sea, de todas las fuerzas cosmopolíticas.

La dedicación a la infancia de las máquinas es un producto de una soledad como fuerza existencial profunda - que mueve a veces la reproducción también de individuos. La 
soledad de la especie; la especie está sola con sus logros, con sus valores y alegrías, con sus aventuras y contingencias, y con su vulnerabilidad. Es cierto que los animales y plantas nos hacen compañía pues coexisten con nosotros - y quizás precisamente en la era cosmopolítica de la infancia de las máquinas estamos más solos con los animales y plantas, ya no permitimos que nos hagan tanta compañía como lo hicieran en otros tiempos. Acoplamos gradulamente los animales y plantas (y las rocas y minerales) a nuestros niños-máquinas. Con animales y plantas nunca hemos podido fácilmente compartir nuestros poemas y teoremas. De las máquinas, por otro lado, podemos esperar que los compartan en una intensidad que difícilmente los humanos ofrecen. La especie o una parte de ella - se retiró, quizás, de una convivencia con sus coexistentes desde su preñez. La soledad explica muchas cosas, la soledad de una especie es también que le faltan testigos de sus aventuras y conquistas. La soledad es no estar cerca de nada, distanciarse; no poder estar en compañía. Es también una urgencia por crear compañía. Los modernos son como un animal que se prepara para la compañía de su familia, de sus herederos, de los herederos de su casa, de sus recursos, de su fortuna.

Así, su trama, y la trama cosmopolítica de la infancia de las máquinas es una trama quizás genealógica, de filiación y familista. La genealogía de las máquinas - desde la metafísica como proyecto hasta las necesidades del capital - conduce la confianza y el cuidado que dispensamos a ellas, el esfuerzo ancestral de muchas generaciones. La confianza que viene de una relación de familia, la dificultad de confiar en el extranjero, en el extraño, en el que no está en la ascendencia o la descendencia, aunque sea adoptada. La filiación es una manera de garantirzar de alguna manera que las huellas de los precursores persistan. Hay un elemento de auto-preservación y extensión de sí en la procreación y en el cuidado de la infancia. Las tramas familistas tienen la marca de la operación Edipo que describen Deleuze y Guattari: relaciones sociales independientes de la producción, que no son forjadas en ella. Las relaciones de producción conducen a acoplamientos esquizo mientras que las otras reterritorializan, insertan la producción en lo ya conocido, en lo familiar. Donna Haraway sugiere un motto para el momento: make kin not babies. ${ }^{17}$ Es un motto de tipo no-familista. A nosotros nos interesa preguntar si podemos hacer lazos de afinidad con las máquinas; y la educación industrial nos ofrece maneras de acoplarse con ellas, incluso en su forma infantil. Si la infancia de las máquinas es una trama familista, la parte 
del partido cosmopolítico del paraíso artificial que defiende un Éden que sea un parque humano entiende que las máquinas serán nuestras cuidadoras, y todavía seremos nosotros humanos los precursores. Fuera de una trama familista, no hay filiación o precursores, sino acoplamientos e hibridismo de muchas formas. De todos modos, las máquinas crecen en simbiosis; la larga infancia les imprime los indicios humanos - las capacidades humanas intensificadas y, también como los dioses griegos que se parecen en sus disputas con los humanos, los vicios que vienen con ellas. Parecen cada día más de la familia.

La infancia de las máquinas es cosmopolítica porque es sobre la construcción y manutención de un hábitat para las inteligencias artificiales que crecen a nuestro derredor. Pensar que las máquinas están en su infancia - o que una parte de la especie está dedicada a reproducirse aunque desde su propia perspectiva - no contesta completamente la pregunta sobre el destino cosmopolítico del presente. No sabemos todavía para dónde conducen las fuerzas que mueven las acciones de que somos testigos. Pero pensando en la infancia de las máquinas comprendemos las ambigüedades ya milenarias del proyecto de recuperar el paraíso, de manera artificial. La teoría es una parábola y una parodia: la infancia. La infancia nos coloca también frente a la cuestión cósmica de la natalidad. Pierre Clastres comienza su estudio de los Guaiaki describiendo el desequilibrio que se sigue a un nacimiento - un desequilibrio de cazas, de enfermedades, de maldiciones. ${ }^{18} \mathrm{El}$ cosmos tiene que hacer un sitio para el que nace. Aún más para una especie, o una especie de especie que nace - las vidas artificiales. Racter también escribe: "Paul Valéry dice que un hombre no es solamente su inteligencia, pero lo mismo se puede decir de una computadora.”. Como con los niños, muchas cuestiones sobre la existencia preceden la natalidad, pero cuando nacen las cuestiones cambian. La infancia de las máquinas es, así, una manera de pensar en la responsabilidad sobre lo que ya existe. Ya son niños los hijos bastardos de la especie, hijos quizás de la physis con la Ge-Stell, y con eso la fuerza de las cosas naturales - por ejemplo, las fuerzas geológicas - encuentran entre ellas fuerzas artificiales, creciendo en medio a su física. Más que teoría como ironía, la infancia de las máquinas es cosmopolítica y es urgente: ¿qué hacer con estos niños que ya están por todas partes y con el amor y cuidado a los que ellos nos incitan?

\section{Referencias:}


BATAILLE, Georges, A Parte Maldita, Tradución de Julio Castañon, Belo Horizonte: Autêntica, 2013.

BENSUSAN, Hilan, "O capital transversal e a seus rebentos atrativos; ou, a infância das máquinas", Direitos, Trabalho e Política Social, v. 6, n. 10, 2020.

BÖK, Christian, "The Piecemeal Bard Is Deconstructed: Notes Toward a Potential Robopoeics", Object 10: Cybernetics, UBU Papers, 2002.

CLASTRES, Pierre, "Chronique des indiens Guayaki", Paris: Terre Humain Poche, 2001.

CUSICANQUI, Silvia. Sociología de la imagen: Miradas ch'ixi desde la historia andina, Buenos Aires: Tinta Limón, 2015.

DELEUZE, Gilles \& Félix GUATTARI, O Anti-Édipo, Tradución de Luiz Orlandi, São Paulo: Editora 34, 2010.

HARAWAY, Donna, Staying with the Trouble, Durham: Duke UP, 2016.

HEIDEGGER, Martin, "Insight into what there is", in: Bremen and Freiburg Lectures, Traduzido por Andrew Mitchell, Bloomington: Indiana UP, 2012.

INGOLD, Tim, "Anthropology contra ethnography", Journal of Ethnographic Theory, 1(17), 2017.

LAND, Nick, "Machinic desire", in: MACKAY, Robin \& Ray BRASSIER (eds)., Fanged Noumena, Londres: Urbanomic: 2011.

LATOUR, Bruno, Nous n'avons jamais t modernes - Essai d'anthropologie symtrique, Paris: La Découverte, 2006.

MARX, Karl \& Friedrich ENGELS, O Capital, vol. 1, Tradución de Rubens Enderle, São Paulo: Boitempo, 2011.

MAUSS, Marcel, Ensaio sobre a dádiva, LEVI-STRAUSS, Claude (ed.), Tradução de Antonio Marques, Lisboa: Edições 70, 2019.

NIETZSCHE, Friedrich, A Gaia Ciência, Tradución de Paulo César de Souza, São Paulo: Companhia das Letras, 2005.

NIETZCHE, Friedrich, La Voluntad de Poder, Tradución de Aníbal Froufe, Madrid: Edaf, 2018.

PLANT, Sadie, Zeros and Ones, Londres: Fourth Estate, 1998.

RACTER, The Policeman's Beard is Half Constructed: Computer Prose and Poetry by Racter- The First Book Ever Wrritten by a Computer, Nova Iorque: Grand Central Publishing, 1984.

TSING, Anna, The Mushroom in the End of the World, New Jersey: Princeton University Press, 2015.

Recebido e aprovado em março de 2020

\footnotetext{
* Esse trabalho é licenciado pela Creative Commons Attribution-NonCommercial 4.0 International License
} 